\title{
The synergistic necrohemorrhagic action of Clostridium perfringens perfringolysin and alpha toxin in the bovine intestine and against bovine endothelial cells
}

\author{
Stefanie Verherstraeten ${ }^{1}$, Evy Goossens ${ }^{1}$, Bonnie Valgaeren², Bart Pardon², Leen Timbermont ${ }^{1}$, Karen Vermeulen ${ }^{1}$,
} Stijn Schauvliege ${ }^{3}$, Freddy Haesebrouck', Richard Ducatelle ${ }^{1}$, Piet Deprez ${ }^{2}$ and Filip Van Immerseel ${ }^{{ }^{*}}$

\begin{abstract}
Bovine necrohemorrhagic enteritis is a major cause of mortality in veal calves. Clostridium perfringens is considered as the causative agent, but there has been controversy on the toxins responsible for the disease. Recently, it has been demonstrated that a variety of $C$. perfringens type A strains can induce necrohemorrhagic lesions in a calf intestinal loop assay. These results put forward alpha toxin and perfringolysin as potential causative toxins, since both are produced by all C. perfringens type A strains. The importance of perfringolysin in the pathogenesis of bovine necrohemorrhagic enteritis has not been studied before. Therefore, the objective of the current study was to evaluate the role of perfringolysin in the development of necrohemorrhagic enteritis lesions in calves and its synergism with alpha toxin. A perfringolysin-deficient mutant, an alpha toxin-deficient mutant and a perfringolysin alpha toxin double mutant were less able to induce necrosis in a calf intestinal loop assay as compared to the wild-type strain. Only complementation with both toxins could restore the activity to that of the wild-type. In addition, perfringolysin and alpha toxin had a synergistic cytotoxic effect on bovine endothelial cells. This endothelial cell damage potentially explains why capillary hemorrhages are an initial step in the development of bovine necrohemorrhagic enteritis. Taken together, our results show that perfringolysin acts synergistically with alpha toxin in the development of necrohemorrhagic enteritis in a calf intestinal loop model and we hypothesize that both toxins act by targeting the endothelial cells.
\end{abstract}

\section{Introduction}

Since the ban on antimicrobial growth promoters in Europe, necrohemorrhagic enteritis emerged as a major cause of mortality in veal calves in Belgium, causing important economic losses [1-3]. Bovine necrohemorrhagic enteritis, also known as enterotoxaemia, is most typically characterized by sudden death and macroscopic postmortem findings are necrotic and hemorrhagic lesions in the small intestine $[4,5]$. Microscopically, necrosis of the intestinal mucosa and hemorrhages are observed $[1,5,6]$. Clostridium perfringens is considered as the

\footnotetext{
* Correspondence: filip.vanimmerseel@ugent.be

'Department of Pathology, Bacteriology and Avian Diseases, Faculty of Veterinary Medicine, Ghent University, Salisburylaan 133, Merelbeke B-9820, Belgium

Full list of author information is available at the end of the article
}

causative agent $[1,7]$. This spore-forming, Gram-positive, anaerobic bacterium is often found as a normal inhabitant of the intestine of most animal species and humans $[6,8,9]$. For reasons that are not yet fully understood, $C$. perfringens can, under certain predisposing conditions, proliferate rapidly, concurrently produce toxins and cause disease. Stress, for example, is considered to be such a predisposing factor, and also the feed composition is believed to be of major importance for the development of the disease $[7,10,11]$. The presence of a causative toxin determines the potential of a C. perfringens strain to cause lesions in several animal species. Classification of $C$. perfringens strains is based on the production of four major toxins, namely alpha, beta, epsilon and iota toxin. In addition to the major toxins,

\section{() Biomed Central}


other toxins can be secreted, such as beta2-toxin and perfringolysin $[5,12]$.

There has been controversy on the toxins responsible for bovine necrohemorrhagic enteritis. Some studies proposed epsilon toxin as a possible causative toxin [13-16]. This toxin is produced by type B and D strains and plays a key role in the pathogenesis of sheep and goat enterotoxaemia. Filho et al. could indeed induce clinical signs and lesions, when a type D strain was inoculated intraduodenally in a calf [15]. In an intestinal loop assay comparing alpha and epsilon toxin, only epsilon toxin was able to cause severe oedema and hemorrhages in the lamina propria [9]. These results point to epsilon toxin as causative toxin. More recently, beta2-toxin (CPB2) has been linked to necrohemorrhagic enteritis in calves and cows [2,6,17-19]. Manteca et al. showed that inoculating a beta2-positive type A strain into a bovine ligated intestinal loop caused hemorrhages of the intestinal wall and necrosis [19]. The strain however also produced high levels of alpha toxin, so a synergistic action between both toxins was proposed. Moreover, allelic variants of the CPB2-gene have been identified and strains from cattle mostly carried the atypical CPB2gene that is not expressed, in contrast to the "consensus" variant $[2,6,17]$. In addition, a more recent study isolated only beta2-negative type A strains from calves with necrohemorrhagic enteritis and these strains were also able to induce pathological changes in inoculated intestinal loops [10]. We recently developed an experimental intestinal loop model that mimics the typical lesions of calf necrohemorrhagic enteritis both macroscopically and microscopically [20]. In this model, it was demonstrated that type A strains from bovine and non-bovine origin, including both beta2-negative and -positive strains, were able to induce necrohemorrhagic lesions [20]. These results suggest that the causative toxin should be present in all the tested $C$. perfringens strains. This puts forward a potential role for alpha toxin and perfringolysin, since both are produced by nearly all $C$. perfringens strains [21]. The importance of perfringolysin in the pathogenesis of bovine necrohemorrhagic enteritis is not reported before.
In gas gangrene or clostridial myonecrosis, perfringolysin is involved in the pathogenesis in synergy with alpha toxin [22-24]. Additionally, Valgaeren et al. observed that the early pathogenesis of calf necrohemorrhagic enteritis in an experimental loop assay is characterized by congestion of the capillaries resulting in hemorrhages and finally necrosis of the mucosa [20]. This points to the endothelium as a possible target.

Therefore, the objective of the present study was to evaluate the role of perfringolysin in the development of necrohemorrhagic enteritis and its synergism with alpha toxin in calves, by using a calf intestinal loop assay and endothelial cell cytotoxicity assays.

\section{Materials and methods}

\section{Bacterial strains and culture conditions}

The bacterial strains used were a wild-type gas gangrene strain (JIR325) and its isogenic mutant genetically deficient in the production of perfringolysin and alpha toxin ( $\triangle p f o A \Delta p l c$ double mutant JIR4444) as well as the $\Delta p f o A$ $\triangle p l c$ double mutant complemented with a plasmid that carried either the perfringolysin gene ( $p f o A$-complemented $\triangle p f o A \Delta p l c$ mutant JIR4460) or the alpha toxin gene (plc-complemented $\triangle p f o A \Delta p l c$ mutant JIR4461) or both toxins ( $p f o A$ plc double-complemented $\triangle p f o A \Delta p l c$ mutant JIR4462) (Table 1) [23,25]. All strains were kindly provided by J.I. Rood (Department of Microbiology, Monash University, Clayton, Victoria, Australia). The strains were grown at $37{ }^{\circ} \mathrm{C}$ in Brain Heart Infusion (BHI) broth (Oxoid, Basingstoke, United Kingdom) with $0.375 \%$ glucose in an anaerobic $\left(84 \% \mathrm{~N}_{2}, 8 \% \mathrm{CO}_{2}\right.$ and $\left.8 \% \mathrm{H}_{2}\right)$ cabinet (Ruskinn Technology, Bridgend, UK). When required, chloramphenicol $(30 \mu \mathrm{g} / \mathrm{mL})$ was added to the growth media. The supernatants were filter-sterilized over a $0.2 \mu \mathrm{M}$ filter before use in the experiments (Merck Millipore, Billerica, Massachusetts). When a logarithmicphase culture was used, a 1/100 dilution from an overnight culture was made $3 \mathrm{~h}$ prior to use and was incubated in an anaerobic cabinet.

Filter-sterilized supernatant of an overnight culture of each strain was assayed for perfringolysin activity by the

Table 1 Description of strains and toxin activities in supernatants of an overnight culture

\begin{tabular}{|c|c|c|c|c|c|c|}
\hline Strain $^{a}$ & $\begin{array}{c}\text { Strain } \\
\text { number }\end{array}$ & Relevant characteristics ${ }^{b}$ & $\begin{array}{l}\text { Plasmid-encoded toxin gene } \\
(\mathbf{s})^{\mathrm{a}}\end{array}$ & $\begin{array}{l}\text { Plc activity } \\
\left.(\mu \mathrm{g} \mathrm{mL})^{-1}\right)^{\mathrm{c}}\end{array}$ & $\begin{array}{l}\text { PFO activity } \\
\left(\log _{2}(\text { titre })\right)^{c}\end{array}$ & Ref. \\
\hline Wild-type & JIR325 & Strain $13^{d}$ & & $12.5 \pm 2.5$ & $4.8 \pm 0.2$ & {$[25]$} \\
\hline$\Delta p f o A \Delta p / c$ & JIR4444 & $\begin{array}{l}\text { JIR325 pfoA:ermB plc@pJIR1774, } \\
\text { suicide plasmid }\end{array}$ & & $<1.0$ & $<1.0$ & {$[23]$} \\
\hline pfoA-complemented $\Delta p f o A \Delta p / c$ & JIR4460 & $J \mid R 4444(p J \mid R 871), C^{R}$ & $p f o A^{+}$ & $<1.0$ & $4.1 \pm 0.3$ & {$[23]$} \\
\hline plc-complemented $\Delta p f o A \Delta p / c$ & $J \mid R 4461$ & $J \mid R 4444(p J I R 1642), \mathrm{Cm}^{R}$ & $p / c^{+}$ & $6.8 \pm 0.7$ & $<1.0$ & [23] \\
\hline $\begin{array}{l}\text { double-complemented } \\
\Delta p f o A \Delta p / c\end{array}$ & JIR4462 & $J \mid R 4444(p J I R 1720), C^{R}$ & $p f o A^{+} p / c^{+}$ & $8.0 \pm 0.5$ & $3.5 \pm 0.1$ & {$[23]$} \\
\hline
\end{tabular}

${ }^{a}$ pfoA: perfringolysin gene, plc : alpha toxin gene, ${ }^{b} \mathrm{Cm}^{\mathrm{r}}$ : chloramphenicol resistant, ${ }^{C}$ Plc : alpha toxin, PFO: perfringolysin $\mathrm{O},{ }^{\mathrm{d}} \mathrm{JIR} 325$ is a rifampicin and nalidixic acid resistant derivative of strain 13 , a gas gangrene strain. 
doubling dilution hemolytic assay with horse red blood cells as described previously (Table 1) [26]. The perfringolysin activity was expressed as the reciprocal of the last dilution which showed complete hemolysis. The perfringolysin assay was performed in triplicate. For the detection of alpha toxin in the supernatant, the Bio$\mathrm{X}$ Alpha Toxin Elisa Kit (Bio-X Diagnostics, Jemelle, Belgium) was used according to the instructions of the manufacturer (Table 1). The amount of alpha toxin was calculated as described previously in $\mu \mathrm{g} \mathrm{mL}^{-1}$ [27]. The alpha toxin ELISA was performed in triplicate.

\section{Assessing the role of perfringolysin by using an intestinal loop model}

The animal studies were undertaken with approval (EC2012_056) of the Ethical Committee of the Faculty of Veterinary Medicine (Ghent University). Three 11week-old Holstein Friesian calves, originating from a commercial veal herd, were used. Intestinal loop assays were conducted as described by Valgaeren et al. [20]. Briefly, the calves were anesthetized and the small intestine was exteriorized. Intestinal loops were ligated and injected with $20 \mathrm{~mL}$ logarithmic cultures followed by an injection of $10 \mathrm{~mL} 25 \%$ commercial milk replacer (Vitaspray, Vitamex ${ }^{\circ}$, Drongen, Belgium) suspended in sterile $0.9 \% \mathrm{NaCl}$ solution. Because the gas gangrene strain JIR325 could induce lesions comparable to the bovine strains, this strain was used in combination with its isogenic mutants (Table 1) [20]. Each strain was injected in quintuplicate and as a control BHI was injected in five loops instead. After injection of the loops, the abdomen was closed and the calves were maintained under anesthesia. At 6-h post-inoculation the animals were euthanized and samples were taken. Samples were fixed in $4 \%$ phosphate buffered formaldehyde. They were embedded in paraffin wax, sectioned and stained with hematoxylin-eosin by the conventional method for histological examination. The sections were evaluated for presence of necrotic lesions (Leica DM L2 microscope with Leica DFC320 camera and LAS software).

\section{Isolation of bovine umbilical vein endothelial cells}

Primary bovine umbilical vein endothelial cells (BUVEC) were isolated from umbilical cord veins by an adaptation of the method of Jaffe et al. [28]. Umbilical cords obtained from calves born by caesarean section, were transported in 0.9\% HBSS-HEPES buffer (pH 7.4, Gibco, Grand Island, NY, USA) supplemented with $500 \mathrm{U} / \mathrm{mL}$ penicillin (Sigma-Aldrich) and $50 \mu \mathrm{g} / \mathrm{mL}$ streptomycin (Sigma-Aldrich). The umbilical vein was cannulated and flushed with pre-warmed $\left(37{ }^{\circ} \mathrm{C}\right)$ HBSS (Gibco, Grand Island, NY, USA). $0.5 \%$ collagenase type IV (Sigma-Aldrich) suspended in HBSS was infused into the lumen of the clamped shut umbilical vein and it was incubated for
30 min at $37{ }^{\circ} \mathrm{C}$. After gently massaging the umbilical vein, the cells were flushed from the vein by perfusion with 10 mL HBSS containing 20\% fetal calf serum (FCS, Bockneck Labs Inc., Toronto, Canada). After rinsing $(250 \times \mathrm{g}, 5 \mathrm{~min})$, a single cell suspension was obtained by filtration through a $70 \mu \mathrm{m}$ cell strainer (BD Labware, San Jose, CA, USA). The pelleted $(250 \times \mathrm{g}, 10 \mathrm{~min})$ cells were resuspended in endothelial cell growth medium containing 20\% FCS (EGM-2; Lonza, Basel, Switzerland) and seeded in $25 \mathrm{~cm}^{2}$ plastic tissue culture flasks. After $24 \mathrm{~h}$ incubation at $37{ }^{\circ} \mathrm{C}$ in the presence of $5 \% \mathrm{CO}_{2}$, the cell medium was changed. Bovine umbilical vein endothelial cells were grown to confluence. The endothelial origin and purity was verified by immunocytochemistry using anti-mouse CD31 antibody (Dako, Heverlee, Belgium).

\section{Assessing the cytotoxic effects of perfringolysin on bovine endothelial cells}

In order to visually assess cytotoxicity, BUVEC were seeded on 13-mm-circular glass slides (VWR International BVBA, Leuven, Belgium) in a 24-well plate at a concentration of $1 \times 10^{5}$ cells $/ \mathrm{mL}$ and were incubated at $37{ }^{\circ} \mathrm{C}$ in the presence of $5 \% \mathrm{CO}_{2}$. After $36 \mathrm{~h}$, the cells were exposed to $3 \%$ filter-sterilized supernatant of an overnight culture diluted in serum-free endothelial cell growth medium (SFM). The strains used for producing the supernatants were the wild-type strain JIR325 and its isogenic mutants (Table 1). SFM was used as a control. After $1.5 \mathrm{~h}$ incubation, the cells were rinsed three times with HBSS containing $\mathrm{Ca}^{2+}$ and $\mathrm{Mg}^{2+}$. They were fixed with 100\% methanol and stained with Haemacolorstain (Merck, Darmstadt, Germany). The glass slides were mounted on a microscope slide and observed microscopically.

To quantify the cytotoxicity, BUVEC were seeded in a 96-well plate at a concentration of $1 \times 10^{5}$ cells $/ \mathrm{mL}$ and incubated for $36 \mathrm{~h}$ as described above. $1 \%$ or $6 \%$ of the above mentioned supernatants in SFM was added to the cells. SFM was used to determine reference values. After $1.5 \mathrm{~h}$ incubation, a Neutral Red Uptake Assay was performed [29]. Briefly, Neutral Red Medium (EGM2 medium containing neutral red (Merck N.V./S.A., Overijse, Belgium)) was added to the cells and the plates were incubated at $37{ }^{\circ} \mathrm{C}$. After $3 \mathrm{~h}$ of incubation, the cells were rinsed with $\mathrm{HBSS}$ containing $\mathrm{Ca}^{2+}$ and $\mathrm{Mg}^{2+}$ and treated with extracting solution (50\% absolute ethanol, $49 \%$ distilled water and $1 \%$ glacial acetic acid) for $15 \mathrm{~min}$ at room temperature on a shaker to extract the neutral red from the cells. The absorbance was determined at $550 \mathrm{~nm}$ and cell viability was expressed as the percentage of viable cells compared to untreated cells (negative control) and cells treated with the supernatant 
of the wild-type (positive control). Each culture supernatant was tested in duplicate in three independent assays.

\section{Statistical analysis}

Significant differences in the number of loops with necrosis between the wild-type and the mutants were determined using a two-tailed Fisher Exact test $(P<$ 0.05). Significant differences between the relative viability of the mutants and $0 \%$, which corresponds with the relative viability of the wild-type strain, were investigated using a two-tailed Wilcoxon signed rank test $(P<0.05)$. A one-way analysis of variance (ANOVA) with the post hoc Tukey-Kramer multiple-comparison test was used to identify significant differences in the relative viability of cells after contact with the supernatants of the mutants $(P<0.001)$. All statistical analyses were performed using GraphPad Prism Software 5.0 (GraphPad Software, Inc., USA).

\section{Results}

Assessing the role of perfringolysin by using an intestinal loop model

Histological examination of loops inoculated with the wild-type strain showed necrosis of the tips of the villi and congestion of the capillaries and hemorrhages in the underlying viable tissue (Figure 1). Rod-shaped bacteria were found attached to cellular debris in the lumen and to the mucosa. To elucidate the relative contribution of perfringolysin and alpha toxin in the induction of necrohemorrhagic enteritis a wild-type strain and its $\triangle p f o A \Delta p l c$ mutant was used in combination with the $\triangle p f o A \Delta p l c$ mutant complemented with either the $p f o A$ or the plc gene or both (Figure 2). Using the same intestinal loop model, Valgaeren et al. observed mucosal hemorrhages, not only in the challenge strain inoculated loops, but also in some control loops. Necrosis only occured after inoculation of a C. perfringens strain [20]. Therefore, in the present study we focussed on the presence of necrosis to compare the mutants with the wildtype strain. Significantly fewer loops injected with the $\triangle p f o A \Delta p l c$ mutant or the $p f o A$-complemented $\triangle p f o A$ $\Delta p l c$ mutant (or plc-deficient strain) showed necrosis in comparison to the wild-type strain $(P<0.01)$. The plccomplemented $\triangle p f o A \Delta p l c$ mutant (or $p f o A$-deficient strain) could induce necrotic lesions in fewer loops as compared to the wild-type strain, but the difference in number of necrotic loops did not reach statistical significance. The double-complemented $\triangle p f o A \Delta p l c$ mutant showed necrosis in as many loops as the wild-type. Apparently, only the complementation of both toxins could restore the activity to that of the wild-type. In the control loops no necrotic lesions were detected.
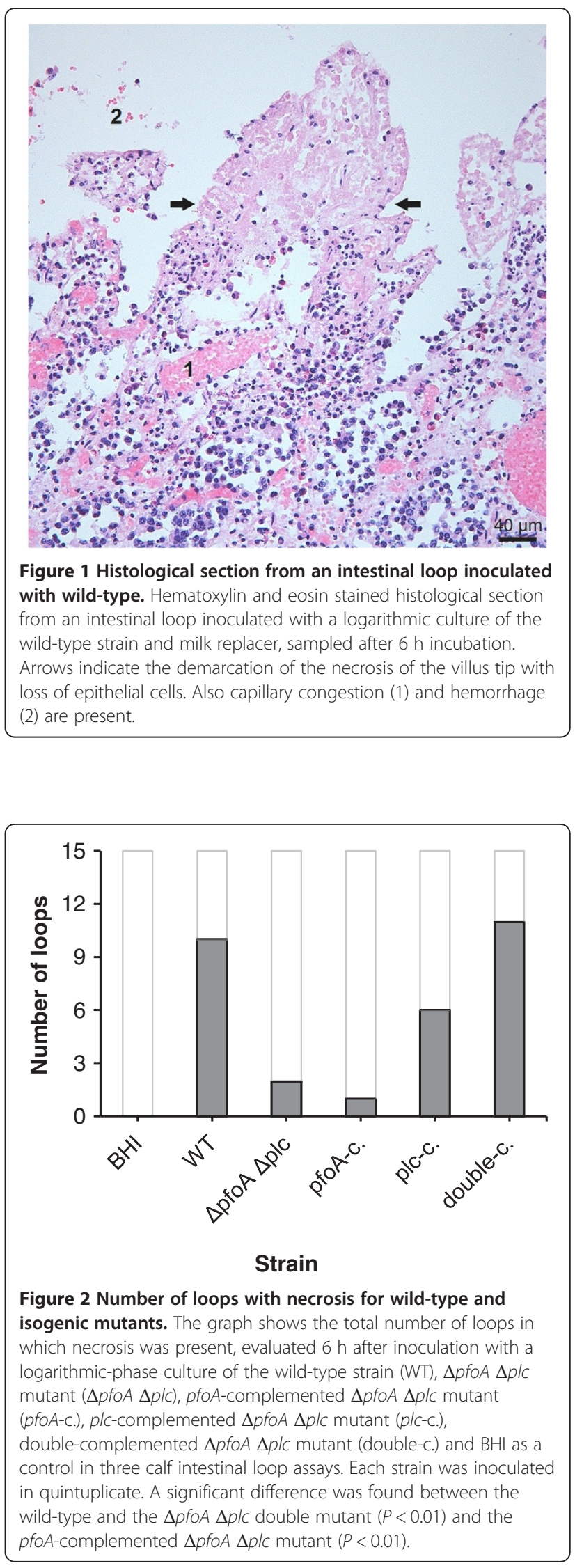


\section{Assessing the cytotoxic effects of perfringolysin on bovine endothelial cells}

To determine whether perfringolysin and alpha toxin have cytotoxic effects on endothelial cells, bovine umbilical cord endothelial cells were exposed to supernatant of a wild-type strain and its isogenic mutants. Almost all cells detached after exposure to supernatant of the wildtype strain (Figure 3 ). No cytotoxicity could be observed visually when using the supernatant of the $\triangle p f o A \Delta p l c$ mutant as compared to the control. The supernatants of the $p f o A$-complemented $\Delta p f o \quad \Delta p l c$ mutant and the plc-complemented $\Delta p l c \Delta p f o$ mutant were less cytotoxic in comparison with supernatant of the wild-type strain, but more cytotoxic as compared to supernatant of the $\Delta p l c \Delta p f o$ mutant. Complementation of the $\Delta p l c \Delta p f_{0}$ mutant with both the $p l c$ and $p f o$ gene restored cytotoxic effects to the wild-type levels. These data indicate that perfringolysin and alpha toxin are important factors for cytotoxicity on bovine endothelial cells and have a synergistic effect.

To quantify the relative contribution of alpha toxin and perfringolysin on endothelial cell cytotoxicity, the viability was assessed by a Neutral Red Uptake Assay. The percentage viability after $1.5 \mathrm{~h}$ incubation with 1 and $6 \%$ supernatant of the isogenic mutants relative to viable untreated cells (negative control) and cells treated with supernatant of the wild-type strain (positive control) are shown in Figure 4. The cytotoxic effect of 6\% supernatant of the $\triangle p f o A \Delta p l c$ mutant was significantly lower than the wild-type $(P<0.05)$ and all other tested strains $(P<0.001)$. The cytotoxicity of $1 \%$ supernatant of $\triangle p f o A \quad \Delta p l c$ mutant, $p f o A$-complemented $\triangle p f o A \quad \Delta p l c$

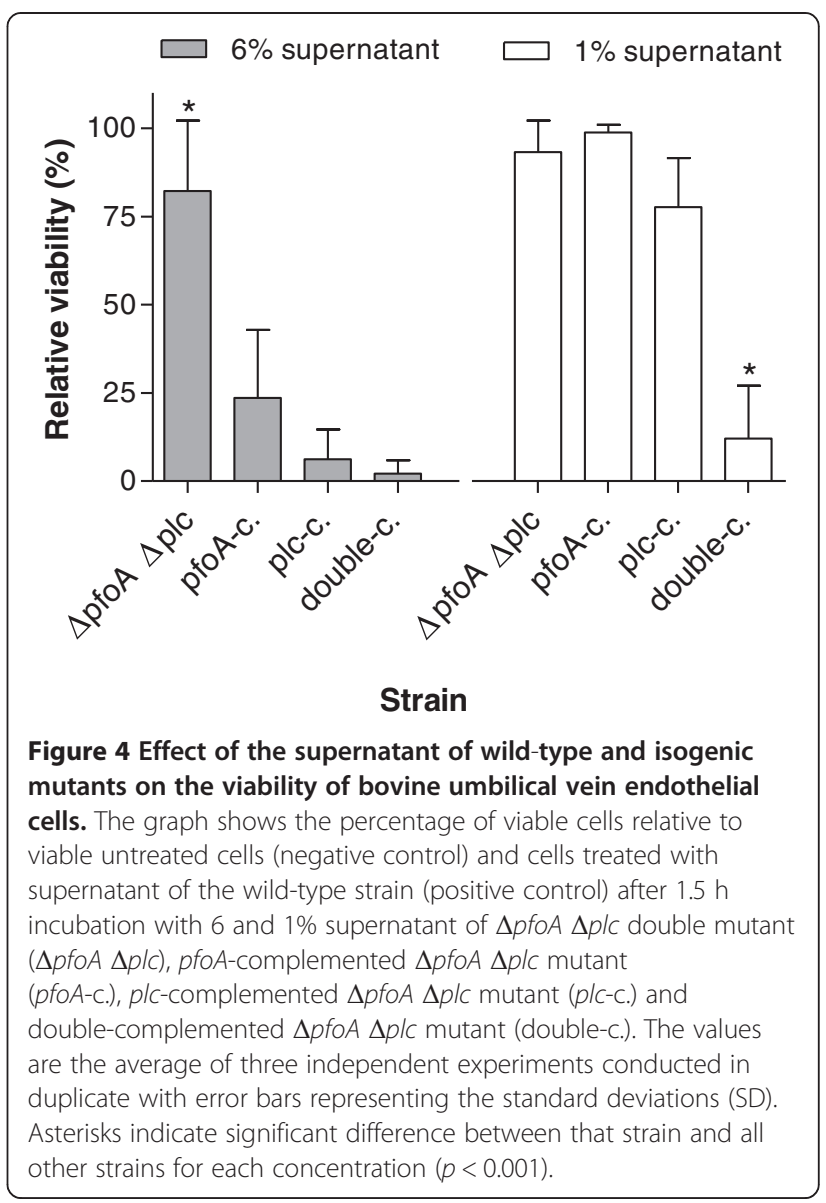

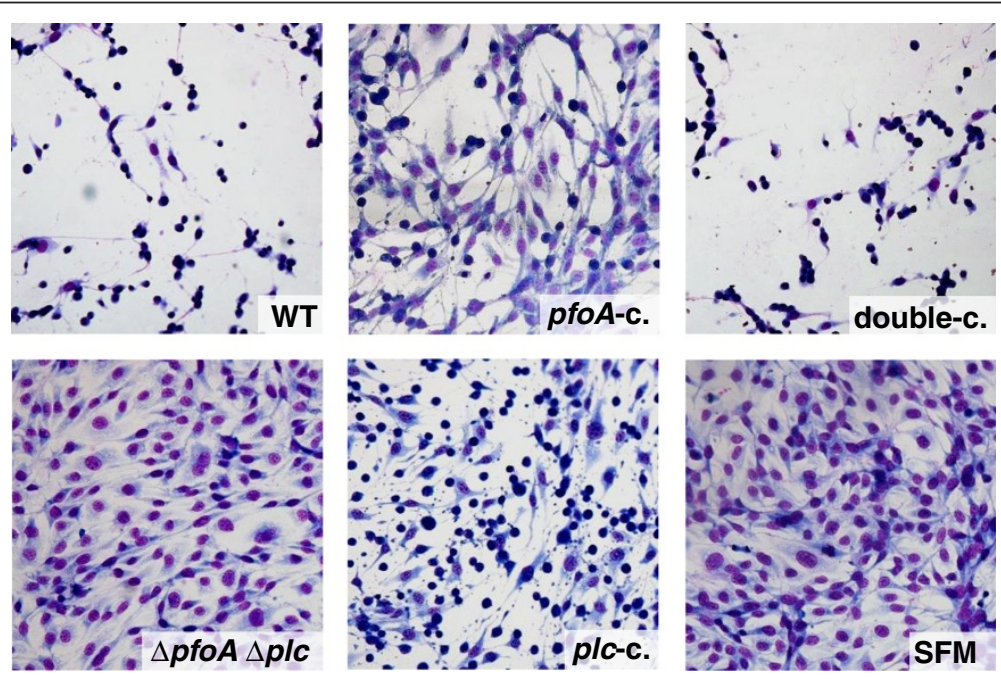

Figure 3 Cytotoxic effect of the supernatant of wild-type and isogenic mutants on bovine umbilical vein endothelial cells (BUVEC.) Photomicrograph of Haemacolor-stained cells after $1.5 \mathrm{~h}$ exposure to $3 \%$ supernatant of the wild-type strain (WT), $\Delta$ pfoA $\Delta p / c$ double mutant $(\Delta p f o A \Delta p / c)$, pfoA-complemented $\Delta p f o A \Delta p / c$ mutant (pfoA-c.), plc-complemented $\Delta p f o A \Delta p / c$ mutant ( $p / c-c$.), double-complemented $\Delta p f \circ A \Delta p / c$ mutant (double-c.) and SFM as a negative control. Magnification, $\times 400$. 
mutant and the plc-complemented $\Delta p f o A \Delta p l c$ mutant was significantly lower as compared to that of the wildtype $(P<0.05)$ and that of the double-complemented $\triangle p f o A \quad \Delta p l c$ mutant $(P<0.001)$. These results confirm that perfringolysin as well as alpha toxin contribute to the cytotoxic effect of a C. perfringens culture, and show a synergistic effect of perfringolysin and alpha toxin.

\section{Discussion}

The presence of a causative toxin in a $C$. perfringens strain determines its potential to cause lesions and subsequently diseases in several animal species. However, there is still controversy on the toxin responsible for bovine necrohemorrhagic enteritis. In a previous study, it was demonstrated that type A strains from bovine and non-bovine origin can induce necrohemorrhagic lesions in a calf intestinal loop assay [20]. These results suggest that the causative toxin is one of the toxins produced by all C. perfringens type A strains. In the present study, it was shown that perfringolysin and alpha toxin are involved in the induction of necrohemorrhagic lesions. Indeed, a plc-complemented $\triangle p f o A \Delta p l c$ mutant (or $p f o A$-deficient strain) and a $p f o A$-complemented $\triangle p f o A$ $\Delta p l c$ mutant (or plc-deficient strain) of a gas gangrene strain had a decreased ability to induce necrohemorrhagic lesions in a calf intestinal loop assay. The gas gangrene strain JIR325 was used, because perfringolysin- and alpha toxin-deficient mutants of this strain were already available and because this strain was able to induce mucosal hemorrhages and necrosis of the villus tips comparable to the lesions induced by bovine strains in a calf intestinal loop model [20]. In previous studies, it was suggested that epsilon toxin and beta2-toxin are essential in the development of necrosis. Filho et al. induced lesions in a calf intraduodenally inoculated with a type D strain [15]. The authors proposed epsilon toxin as causative toxin. However, based on our results, alpha toxin and perfringolysin may also have been involved in lesion development, since both toxins are produced by type D strains as well. In an intestinal loop assay, comparing alpha and epsilon toxin, only epsilon toxin was able to cause severe oedema and hemorrhages in the lamina propria [9], but these authors did not observe necrosis, in contrast to our results which showed necrohemorrhagic lesions comparable to field cases. Manteca et al. stated that beta2-toxin was an essential toxin, because inoculation of a beta2-positive type A strain into a bovine ligated intestinal loop caused necrohemorrhages of the intestinal wall [19]. However, the strain also produced a high level of alpha toxin and the authors proposed a synergistic effect of alpha toxin and beta2-toxin. Since nearly all type A strains produce perfringolysin as well, this toxin may also have been important in the development of the lesions. In addition, our results agree with a recent study in which only beta2- negative type A strains were isolated from calves with necrotic enteritis and these strains were able to induce pathologic changes when inoculated in intestinal loops [10]. It is still possible that beta2-toxin or other toxins can have supplementary effects.

Valgaeren et al. also found indications that endothelial damage may be involved in the early stages of intestinal lesion development [20]. Our results suggest perfringolysin-induced cytotoxic effects on endothelial cells may play a potential role in the development of necrohemorrhagic enteritis. We showed that the plccomplemented $\triangle p f o A \Delta p l c$ mutant (or $p f o A$-deficient strain) was significantly less cytotoxic for bovine umbilical cord endothelial cells (BUVEC). Endothelial cells form a vital barrier that controls the exchange of cells, macromolecules and fluids between the vascular lumen and the surrounding tissue. They also maintain the normal blood flow due to their antiplatelet, anticoagulant and fibrinolytic properties. Disruption of the endothelial barrier leads to increased vascular permeability along with tissue edema and hemorrhage. Furthermore, it augments local coagulation and vascular thrombosis, and subsequent hypoxic tissue necrosis [30]. In order to confirm that the endothelium is the target cell of perfringolysin or alpha toxin in bovine necrohemorrhagic enteritis, it would be interesting to localize the toxin in lesions, as already been done for beta toxin in necrotic enteritis in piglets and in a human case $[31,32]$. Beta toxin has been shown to induce porcine endothelial cell damage in vitro and to bind to endothelial cells, and not to epithelial cells, in the gut of diseased animals, suggesting that disruption of endothelial cells plays a role in type C enteritis [30,31,33].

Additionally, our results show that perfringolysin and alpha toxin act synergistically in inducing BUVEC cytotoxicity and necrohemorrhagic lesions in a calf intestinal loop model. In gas gangrene, perfringolysin and alpha toxin also act synergistically [22-24,34]. Alpha toxin is a phospholipase $\mathrm{C}$ that hydrolyzes phosphatidylcholine and sphingomyelin, both of which are important constituents of eukaryotic cell membranes. Perfringolysin is a cholesterol-dependent cytolysin and oligomerizes upon contact with cholesterol-containing membranes to form large transmembrane pores by inserting a beta-barrel into the membrane [24]. It has been stated that the ability of perfringolysin to perforate the membrane of target cells, is determined by the amount of free cholesterol molecules present $[35,36]$. Moe and Heuck found that alpha toxin cleaves the phosphocholine headgroup of phosphatidylcholine, increasing the number of free cholesterol molecules in the membrane and by doing so, facilitating the interaction of perfringolysin and cholesterol [36]. This concerted action of alpha toxin and perfringolysin may contribute to the synergistic effect 
between both toxins in gas gangrene and in bovine necrohemorrhagic enteritis.

In gas gangrene, perfringolysin modulates the host inflammatory response by upregulating leukocyte and endothelial adhesion molecules. This causes leukocyte accumulation within the blood vessels and inhibits the normal influx of phagocytic cells into infected host tissue, reducing inflammation [22,23,37]. Additionally, alpha toxin enhances the expression of platelet adhesion molecules, contributing to the formation of freely moving intravascular aggregates of platelets, fibrin and neutrophils. This leads to the obstruction of the vessels and contributes to a decreased blood flow [34]. Gas gangrene is characterized by tissue necrosis, thrombosis and a lack of leukocyte infiltration at the site of infection. On the contrary, bovine necrohemorrhagic enteritis is associated with congestion of the capillaries, hemorrhages and inflammation $[7,10,20]$. So perfringolysin and alpha toxin appear to be involved in both diseases, but may act in a different way. The use of mutants deficient in the production of alpha toxin or perfringolysin in a mouse myonecrosis model showed that alpha toxin is essential for thrombosis formation [22,23]. Furthermore, when rabbits were treated intravenously with recombinant perfringolysin a vasodilatory effect and a reduced systemic vascular resistance was observed. On the other hand, in rabbits treated with recombinant alpha toxin the vascular resistance was maintained and the arterial pressure was reduced $[23,38,39]$. Altered vascular integrity, but not vascular occlusion, seems to be in accordance with the role of perfringolysin in bovine necrohemorrhagic enteritis. This may also explain partly the inflammation present in necrohemorrhagic enteritis as opposed to the lack of leukocyte infiltration in gas gangrene.

Next to the toxic effect of perfringolysin on endothelial cells, other effects on the gastro-intestinal mucosa are most likely of importance in the development of necrohemorrhagic enteritis. Indeed, before perfringolysin can target the endothelial cells, it has to cross the epithelial barrier. While cytotoxic effects of perfringolysin and alpha toxin on intestinal epithelial cells cannot be excluded, also other C. perfringens toxins, enzymes or other molecules could affect the intestinal integrity. Intestinal integrity disturbances can also be caused by $C$. perfringens independent factors in the field, such as viral and parasitological pathogens. The most well-known example of a predisposing pathogen for necrotic enteritis is coccidiosis in broilers [40,41], but also in calves several infectious agents, such as coccidia, enteropathogenic bacteria, corona- and rotaviruses can affect the intestinal barrier integrity [40-44]. In addition, certain feed components can act as predisposing factors for the induction of gut lesions. These include high non-starch polysaccharide containing diets and high protein diets, the latter most likely feeding the auxotrophy $C$. perfringens has for many amino acids $[45,46]$. The diet can have a direct effect on the virulence of $C$. perfringens, but it can as well affect the intestinal tract. In calves fed with milk replacing proteins an increase in permeability of the intestinal mucosa was observed, which caused leakage of macromolecules from the gut into the tissues [41,47-49]. This might also facilitate the uptake of toxins through the epithelial barrier.

In conclusion, our study indicates that perfringolysin is involved in the pathogenesis of bovine necrohemorrhagic enteritis and acts synergistically with alpha toxin. We hypothesize that both toxins may induce intestinal lesions by targeting the endothelial cells.

\section{Competing interests}

The authors declare that they have no competing interests.

\section{Authors' contributions}

SV, EG, BV, BP, LT, RD, PD and FVI participated in the design of the study. SV, $E G, B V, B P, K V, S S$ and RD performed the calf intestinal loop assays. SV carried out the cell cytotoxicity assays and analyzed the data. SV, FH, RD, PD and FVI wrote the manuscript. All authors revised the manuscript and read and approved the final manuscript.

\section{Acknowledgements}

The authors thank J.I. Rood for providing the bacterial strains used in this study. The authors acknowledge support from all veterinary surgeons from the division of anesthesia for their assistance in the intestinal loop assays, the PhD students from the pathology-department who assisted in the sampling and the department of obstetrics, reproduction and herd health for the supply of bovine umbilical cords. The authors would like to thank Christian Puttevils, Delphine Ameye and Astra Dhanijns for the technical assistance. This project was supported by the Institute for Science and Technology, Flanders under contract number 090910.

\section{Author details}

${ }^{1}$ Department of Pathology, Bacteriology and Avian Diseases, Faculty of Veterinary Medicine, Ghent University, Salisburylaan 133, Merelbeke B-9820, Belgium. ${ }^{2}$ Department of Internal Medicine and Clinical Biology of Large Animals, Faculty of Veterinary Medicine, Ghent University, Salisburylaan 133, Merelbeke B-9820, Belgium. ${ }^{3}$ Department of Surgery and Anesthesia of Domestic Animals, Faculty of Veterinary Medicine, Ghent University, Salisburylaan 133, Merelbeke B9820, Belgium.

Received: 15 April 2013 Accepted: 7 June 2013

Published: 19 June 2013

\section{References}

1. Manteca C, Daube G, Pirson V, Limbourg B, Kaeckenbeeck A, Mainil JG Bacterial intestinal flora associated with enterotoxaemia in Belgian Blue calves. Vet Microbiol 2001, 81:21-32.

2. Muylaert A, Lebrun M, Duprez JN, Labrozzo S, Theys H, Taminiau B, Mainil J: Enterotoxaemia-like syndrome and Clostridium perfringens in veal calves. Vet Rec 2010, 167:64-65.

3. Pardon B, De Bleecker K, Hostens M, Callens J, Dewulf J, Deprez P: Longitudinal study on morbidity and mortality in white veal calves in Belgium. BMC Vet Res 2012, 8:26.

4. Valgaeren BR, Pardon B, Verherstraeten S, Goossens E, Timbermont L, Haesebrouck F, Ducatelle R, Deprez PR, Van Immerseel F: Intestinal clostridial counts have no diagnostic value in the diagnosis of enterotoxaemia in veal calves. Vet Rec 2013, 172:237.

5. Songer JG: Clostridial enteric diseases of domestic animals. Clin Microbiol Rev 1996, 9:216-234.

6. Lebrun M, Filee P, Mousset B, Desmecht D, Galleni M, Mainil JG, Linden A: The expression of Clostridium perfringens consensus beta2 toxin is associated with bovine enterotoxaemia syndrome. Vet Microbio/ 2007, 120:151-157. 
7. Lebrun M, Mainil JG, Linden A: Cattle enterotoxaemia and Clostridium perfringens: description, diagnosis and prophylaxis. Vet Rec 2010, 167:13-22.

8. Rood Jl: Virulence genes of Clostridium perfringens. Annu Rev Microbiol 1998, 52:333-360.

9. Morris WE, Dunleavy MV, Diodati J, Berra G, Fernandez-Miyakawa ME: Effects of Clostridium perfringens alpha and epsilon toxins in the bovine gut. Anaerobe 2012, 18:143-147.

10. Morris WE, Venzano AJ, Elizondo A, Vilte DA, Mercado EC, Fernandez-Miyakawa ME: Necrotic enteritis in young calves. J Vet Diagn Invest 2011, 23:254-259.

11. Nowell VJK, Kropinsky AM, Songer JG, Macinnes JI, Parreira VR, Prescott JF: Genome sequencing and analysis of a type A Clostridium perfringens isolate from a case of bovine clostridial abomasitis. PLoS One 2012, 7:e32271.

12. Songer JG, Miskimmins DW: Clostridium perfringens type E enteritis in calves: two cases and a brief review of the literature. Anaerobe 2004 10:239-242.

13. Niilo L, Avery RJ: Bovine "enterotoxemia" I. Clostridium Perfringens types isolated from animal sources in Alberta and Saskatchewan. Can Vet $J$ 1963, 4:31-36

14. Uzal FA, Kelly WR, Morris WE, Assis RA: Effects of intravenous injection of Clostridium perfringens type D epsilon toxin in calves. J Comp Pathol 2002, 126:71-75.

15. Filho EJ, Carvalho AU, Assis RA, Lobato FF, Rachid MA, Carvalho AA, Ferreira PM, Nascimento RA, Fernandes AA, Vidal JE, Uzal FA: Clinicopathologic features of experimental Clostridium perfringens type $D$ enterotoxemia in cattle. Vet Pathol 2009, 46:1213-1220.

16. Uzal FA, Rolfe BE, Smith NJ, Thomas AC, Kelly WR: Resistance of ovine, caprine and bovine endothelial cells to Clostridium perfringens type $D$ epsilon toxin in vitro. Vet Res Commun 1999, 23:275-284.

17. Gibert M, Jolivet-Reynaud C, Popoff MR: Beta2 toxin, a novel toxin produced by Clostridium perfringens. Gene 1997, 203:65-73.

18. Bueschel DM, Jost BH, Billington SJ, Trinh HT, Songer JG: Prevalence of $\mathrm{cpb} 2$, encoding beta2 toxin, in Clostridium perfringens field isolates: correlation of genotype with phenotype. Vet Microbiol 2003, 94:121-129.

19. Manteca C, Daube G, Jauniaux T, Linden A, Pirson V, Detilleux J, Ginter A Coppe $P$, Kaeckenbeeck A, Mainil JG: A role for the Clostridium perfringens beta2 toxin in bovine enterotoxaemia? Vet Microbiol 2002, 86:191-202.

20. Valgaeren B, Pardon B, Goossens E, Verherstraeten S, Schauvliege S, Timbermont L, Ducatelle R, Deprez P, Van Immerseel F: Lesion development in a new intestinal loop model indicates the involvement of a shared Clostridium perfringens virulence factor in haemorrhagic enteritis in calves. J Comp Pathol 2013, 149:103-112.

21. Rood J, Cole ST: Molecular genetics and pathogenesis of Clostridium perfringens. Microbiol Rev 1991, 55:621-648

22. Ellemor DM, Baird RN, Awad MM, Boyd RL, Rood Jl, Emmins JJ: Use of genetically manipulated strains of Clostridium perfringens reveals that both alpha-toxin and theta-toxin are required for vascular leukostasis to occur in experimental gas gangrene. Infect Immun 1999, 67:4902-4907.

23. Awad MM, Ellemor DM, Boyd RL, Emmins JJ, Rood Jl: Synergistic effects of alpha-toxin and perfringolysin $\mathrm{O}$ in Clostridium perfringens-mediated gas gangrene. Infect Immun 2001, 69:7904-7910.

24. O'Brien DK, Melville SB: Effects of Clostridium perfringens alpha-toxin (PLC) and perfringolysin $\mathrm{O}$ (PFO) on cytotoxicity to macrophages, on escape from the phagosomes of macrophages, and on persistence of $C$. perfringens in host tissues. Infect Immun 2004, 72:5204-5215.

25. Lyristis M, Bryant AE, Sloan J, Awad MM, Nisbet IT, Stevens DL, Rood JI: Identification and molecular analysis of a locus that regulates extracellular toxin production in Clostridium perfringens. Mol Microbiol 1994, 12:761-777.

26. Awad MM, Bryant AE, Stevens DL, Rood Jl: Virulence studies on chromosomal alpha-toxin and theta-toxin mutants constructed by allelic exchange provide genetic evidence for the essential role of alpha-toxin in Clostridium perfringens-mediated gas gangrene. Mol Microbiol 1995, 15:191-202.

27. Zhang G, Darius S, Smith SR, Ritchie SJ: In vitro inhibitory effect of hen egg white lysozyme on Clostridium perfringens type A associated with broiler necrotic enteritis and its alpha-toxin production. Lett App/ Microbiol 2006, 42:138-143.

28. Jaffe EA, Nachman RL, Becker CG, Minick CR: Culture of human endothelial cells derived from umbilical veins. Identification by morphologic and immunologic criteria. J Clin Invest 1973, 52:2745-2756.
29. Van Parys A, Boyen F, Verbrugghe E, Leyman B, Bram F, Haesebrouck F, Pasmans F: Salmonella Typhimurium induces SPI-1 and SPI-2 regulated and strain dependent downregulation of MHC II expression on porcine alveolar macrophages. Vet Res 2012, 43:52.

30. Gurtner C, Popescu F, Wyder M, Sutter E, Zeeh F, Frey J, Von Schubert C, Posthaus H: Rapid cytopathic effects of Clostridium perfringens beta-toxin on porcine endothelial cells. Infect Immun 2010, 78:2966-2973.

31. Miclard J, Jäggi $M$, Sutter $E$, Wyder M, Grabscheid B, Posthaus H: Clostridium perfringens beta-toxin targets endothelial cells in necrotizing enteritis in piglets. Vet Microbiol 2009, 137:320-325

32. Miclard J, Van Baarlen J, Wyder M, Grabscheid B, Posthaus H: Clostridium perfringens $\beta$-toxin binding to vascular endothelial cells in a human case of enteritis necroticans. J Med Microbiol 2009, 58:826-828.

33. Schumacher VL, Martel A, Pasmans F, Van Immerseel F, Posthaus $\mathrm{H}$ : Endothelial binding of beta toxin to small intestinal mucosal endothelial cells in early stages of experimentally induced Clostridium perfringens type $C$ enteritis in pigs. Vet Pathol, in press.

34. Hickey MJ, Kwan RY, Awad MM, Kennedy CL, Young LF, Hall P, Cordner LM, Lyras D, Emmins JJ, Rood Jl: Molecular and cellular basis of microvascular perfusion deficits induced by Clostridium perfringens and Clostridium septicum. PLoS Pathog 2008, 4:e1000045.

35. Flanagan JJ, Tweten RK, Johnson AE, Heuck AP: Cholesterol exposure at the membrane surface is necessary and sufficient to trigger perfringolysin O binding. Biochemistry 2009, 48:3977-3987.

36. Moe PC, Heuck AP: Phospholipid hydrolysis caused by Clostridium perfringens alpha-toxin facilitates the targeting of perfringolysin $\mathrm{O}$ to membrane bilayers. Biochemistry 2010, 49:9498-9507.

37. Stevens DL, Tweten RK, Awad MM, Rood JI, Bryant AE: Clostridial gas gangrene: evidence that alpha and theta toxins differentially modulate the immune response and induce acute tissue necrosis. J Infect Dis 1997, 176:189-195.

38. Asmuth DM, Olson RD, Hackett SP, Bryant AE, Tweten RK, Tso JY, Zollman T, Stevens DL: Effects of Clostridium perfringens recombinant and crude phospholipase $C$ and theta-toxin on rabbit hemodynamic parameters. $J$ Infect Dis 1995, 172:1317-1323.

39. Stevens DL, Bryant AE: The role of clostridial toxins in the pathogenesis of gas gangrene. Clin Infect Dis 2002, 35:S93-S100.

40. Drew MD, Syed NA, Goldade BG, Laarveld B, Van Kessel AG: Effects of dietary protein source and level on intestinal populations of Clostridium perfringens in broiler chickens. Poult Sci 2004, 83:414-420.

41. Van Immerseel F, De Buck J, Pasmans F, Huyghebaert G, Haesebrouck F, Ducatelle R: Clostridium perfringens in poultry: an emerging threat for animal and public health. Avian Pathol 2004, 33:537-549.

42. Lindsay DS, Dubey JP, Fayer R: Extraintestinal stages of Eimeria bovis in calves and attempts to induce relapse of clinical disease. Vet Parasitol 1990, 36:1-9.

43. Chase CC, Hurley DJ, Reber AJ: Neonatal immune development in the calf and its impact on vaccine response. Vet Clin North Am Food Anim Pract 2008, 24:87-104

44. Wei S, Gong Z, Che T, Guli A, Tian F: Genotyping of calves rotavirus in China by reverse transcription polymerase chain reaction. J Virol Methods 2013, 189:36-40.

45. Boyd MJ, Logan MA, Tytell AA: The growth requirements of Clostridium perfringens (welchii) BP6K. J Biol Chem 1948, 174:1013-1025.

46. Fuchs AR, Bonde GJ: The nutritional requirements of Clostridium perfringens. J Gen Microbiol 1957, 16:317-329.

47. Barratt ME, Strachan PJ, Porter P: Antibody mechanisms implicated in digestive disturbances following ingestion of soya protein in calves and piglets. Clin Exp Immunol 1978, 31:305-312.

48. Kilshaw PJ, Slade H: Passage of ingested protein into the blood during gastrointestinal hypersensitivity reactions: experiments in the preruminant calf. Clin Exp Immunol 1980, 41:575-582.

49. Silva AG, Huber JT, Herdt TH, Holland R, Degregorio RM, Mullaney TP: Morphological alterations of small intestinal epithelium of calves caused by feeding soybean protein. J Dairy Sci 1986, 69:1387-1393.

doi:10.1186/1297-9716-44-45

Cite this article as: Verherstraeten et al.: The synergistic

necrohemorrhagic action of Clostridium perfringens perfringolysin and alpha toxin in the bovine intestine and against bovine endothelial cells. Veterinary Research 2013 44:45. 